\title{
Ring chromosome 7 syndrome
}

INSERM

\section{Source}

INSERM. (1999). Orphanet: an online rare disease and orphan drug data base. Ring chromosome 7 syndrome. ORPHA:1449

Ring chromosome 7 syndrome is a rare chromosomal anomaly syndrome, with highly variable phenotype, principally characterized by growth failure, short stature, intellectual disability, dermatological abnormalities (nevus flammeus, dark pigmented nevi, café-aulait spots), microcephaly and facial dysmorphism (incl. facial asymmetry, small ears, abnormal palpebral fissures, ptosis, epicanthic folds, hyper/hypotelorism). Additional reported features include convulsions, cleft lip and palate, clinodactyly, kyphoscoliosis and genital anomalies (i.e. cryptorchidism, hypospadias, micropenis). 